\title{
Endoscopic and clinical evaluation of treatment and prognosis of Cronkhite-Canada syndrome: a Japanese nationwide survey
}

\author{
Chikako Watanabe ${ }^{1} \cdot$ Shunsuke Komoto $^{1} \cdot$ Kengo Tomita $^{1} \cdot$ Ryota Hokari $^{1}$ • \\ Masanori Tanaka ${ }^{2} \cdot$ Ichiro Hirata $^{3} \cdot$ Toshifumi Hibi $^{4} \cdot$ Jonathan D. Kaunitz $^{5}$. \\ Soichiro Miura ${ }^{1}$
}

Received: 20 May 2015/Accepted: 13 July 2015/Published online: 28 July 2015

(C) The Author(s) 2015. This article is published with open access at Springerlink.com

\begin{abstract}
Background First reported in 1955, Cronkhite-Canada syndrome (CCS), a rare syndrome characterized by ectodermal abnormalities and inflammatory changes of the gastrointestinal tract mucosa, has been associated with a poor prognosis and life-threatening malignant complications. In a large population survey, we endeavored to characterize the course and treatment outcome of CCS through clinical and endoscopic assessment, and to explore its optimal treatment and surveillance strategy.

Methods A retrospective analysis of 210 patients with CCS was conducted via a questionnaire-based nationwide survey of 983 teaching hospitals located throughout Japan. We assessed clinical features, endoscopic findings, treatments used, and short- and long-term outcomes.
\end{abstract}

Electronic supplementary material The online version of this article (doi:10.1007/s00535-015-1107-7) contains supplementary material, which is available to authorized users.

Chikako Watanabe

chikakow@ndmc.ac.jp

1 Department of Internal Medicine, National Defense Medical College, 3-2 Namiki, Tokorozawa, Saitama 359-8513, Japan

2 Department of Pathology and Laboratory Medicine, Hirosaki City Hospital, Aomori, Japan

3 Department of Gastroenterology, Fujita Health University, Aichi, Japan

4 Center for Advanced IBD Research and Treatment, Kitasato Institute Hospital, Tokyo, Japan

5 Greater Los Angeles VA Medical Center and Department of Medicine and Department of Surgery, David Geffen School of Medicine, University of California, Los Angeles,

Los Angeles, CA, USA
Results The average age at diagnosis was 63.5 years. In all cases, upper or lower gastrointestinal tract polyposis was confirmed, accompanied by characteristic ectodermal abnormalities. Of the treatments used, oral corticosteroids (30-49 mg/day) were the most effective treatment for active disease, with adjunctive nutritional support considered beneficial. With corticosteroid treatment, abdominal symptoms were relieved within a few months, whereas polyp regression often required more than 6 months. Maintenance of endoscopic remission with or without steroids for 3 years significantly lowered the development of CCS-related cancer, compared with relapsers or nonresponders, underscoring the importance of sustained endoscopic remission for cancer prevention.

Conclusions The prognosis of CCS has greatly improved through the use of improved medical treatment. Although CCS continues to be relentlessly progressive, carrying a high cancer risk, a sufficient dose and duration of corticosteroid therapy accompanied by nutritional support and periodic endoscopic surveillance appears to improve its natural history.

Keywords Acquired gastrointestinal polyposis syndrome - Malnutrition · Gastric cancer - Colon cancer

\section{Introduction}

Cronkhite-Canada syndrome (CCS) is an uncommon gastrointestinal polyposis syndrome characterized by dermatologic manifestations associated with chronic diarrhea, malnutrition, and enteric protein wasting due to chronic inflammatory changes of the intestinal mucosa [1-5]. The risk of colon cancer in CCS patients may be elevated owing to difficulties in detecting premalignant adenomas in the 
face of multiple inflammatory pseudopolyps. Since it was first reported by Cronkhite and Canada [6], more than 400 cases of CCS have been reported worldwide, with $75 \%$ reported from Japan $[1,3,7-10]$. Given the rarity of CCS, current understanding is based on anecdotal reports and small case series, [10, 11], with no published systematic investigations based on endoscopic features. CCS is sometimes difficult to diagnose for clinicians and pathologists alike, owing to the considerable overlap of histologic and clinical features shared among CCS-related, juvenile, and inflammatory polyps [3, 12-14]. Its clinical course is characterized by progressive disease with occasional spontaneous remissions and frequent relapses [15-18], with rare progression to malignancy $[1,19,20]$. Concomitant cancers occurring in the gastrointestinal tract have been reported, although no systemic investigation of the cancer risk and prognosis has been published since the 1980s [11, 19-23]. The mortality of CCS can be up to $50 \%$ if it is untreated or if treatment is delayed or inadequate [11]. Nevertheless, no treatment in use to date is based on systematically acquired data based on the study of a large population of CCS patients.

Current CCS treatments used individually or in combination include steroids $[1,11,17,18,24]$, nutritional therapy [25], 5-aminosalicylate acid [26], histamine $\mathrm{H}_{2}$ receptor antagonists [27], anti-tumor necrosis factor $\alpha$ (TNF- $\alpha$ ) agents [28], immunomodulators [28], and eradication of Helicobacter pylori [29]. Steroids are considered the mainstay of medical treatment, although the recommended dose and duration of their use have varied widely in the literature, with no current "gold standard" $[11,17$, $18,30]$. Since symptom relapse is common during a steroid taper, a steroid-sparing strategy is needed.

The risk of gastrointestinal cancer may warrant aggressive screening of CCS patients. Since it is nearly impossible to endoscopically detect malignant polyps or concurrent adenocarcinoma given the myriad of inflammatory-type polyps in CCS patients, an evidence-based surveillance program is needed. Therefore the aims of this study were (1) to elucidate the endoscopic characteristics, long-term prognosis, and cancer risk of CCS, and (2) to identify whether corticosteroid therapy can improve primary and long-term outcomes, and if so, what dose and duration are optimal, and (3) to suggest the ideal endoscopic surveillance schedule for CCS, by the use of data from a nationwide survey in Japan.

\section{Materials and methods}

A questionnaire-based nationwide survey of CCS was conducted in 983 Japanese teaching hospitals specializing in gastroenterology. To obtain the most accurate endoscopic information, clinical and endoscopic data were collected from hospitals registered with the Japanese Society of Gastroenterology. The preliminary survey asked: "Has your institute treated patients with Cronkhite-Canada syndrome? If yes, how many?" Responses were received from 591 of 983 facilities $(60.1 \%)$, revealing 213 patients with CCS were treated in 140 facilities between 2000 and 2013. A detailed survey was sent to these facilities, with information obtained from 139 facilities describing 210 patients. The detailed survey consisted of 133 question items organized into 11 sections requesting detailed clinical features, including endoscopic findings, polyp pathology, response to treatment, and prognosis, with an emphasis on the risk of developing gastrointestinal cancer.

Demographic and explicit clinical, laboratory, and endoscopic data were collected at the time of diagnosis. Clinical symptoms such as diarrhea and ectodermal abnormalities, and laboratory tests including testing for protein-losing enteropathy, autoimmunity, or $H$. pylori infection were documented. Endoscopic features included the distribution pattern and size of polyps, and the mucosal appearance of polyps, noting if they appear to be friable, edematous, or engorged [31]. Histological features such as gland dilation or crypt distortion such as branching or withering and the degree of inflammatory cell infiltration were noted.

Treatment outcome was evaluated in two stages. Early outcome was evaluated 6-12 months after the start of treatment or initial diagnosis. Treatment outcome was assessed primarily on the basis of endoscopic improvement of mucosal findings. Early outcomes were classified into "good response" and "no-response" groups. "Good early response" was defined as partial regression of polyposis with mild friability or decreased erythema. "Endoscopic remission" was defined as the absence of polyposis with normal appearance of mucosa, accompanied by a clinical response. "No early response" was defined as no significant endoscopic improvement irrespective of clinical response. "Clinical response" was defined as regression of clinical symptoms accompanied by normalization of the serum albumin concentrations (clinical remission).

The subsequent outcome was evaluated over a 3-year period after initial induction treatment with corticosteroids had started, and patients were classified into "long-term response" and "no long-term response" groups. "Longterm response" includes the maintenance of complete remission and partial remission. "Complete remission" was defined as endoscopic and clinical remission without ongoing medical treatment. "Partial remission" was defined as near endoscopic remission accompanied by clinical remission while the patient was receiving stable corticosteroid therapy at less than $10 \mathrm{mg}$ /day for more than 1 year. "No long-term response" includes episodically 
active disease and clinically chronic active disease without a long-lasting endoscopic remission or improvement. In episodic active clinical disease, frequent use of corticosteroids was necessary to prevent endoscopic relapse. In chronic active disease, sustained endoscopic improvement was not achieved, even with long-term, high-dose corticosteroid therapy. The $\chi^{2}$ test was used to compare two discrete variables, whereas the nonparametric test was used to compare discrete and a continuous variable. Significance was taken as $5 \%$. The study was approved by the Ethics Committee of the National Defense Medical College.

\section{Results}

Of 210 patients with CCS, 136 were men and 74 were women, yielding a male-to-female ratio of 1.84:1. The mean age of onset of symptomatic disease was 63.5 (31-86) years. The observation period was $5.93 \pm 4.85$ (0.5-28) years. All patients were Japanese, except for two non-Japanese Asian women. There was no evidence of polyposis in any blood relatives. Of the 210 patients, 33 $(15.7 \%)$ had died at the time of the survey. Of the 33 deaths, ten were disease-related (three patients died of gastric cancer, three patients died of colon cancer, and four patients died of sepsis caused by colitis and pancreatitis), whereas the other 23 patients died of illness presumed to be unrelated to the primary disease (e.g., lung cancer, cerebral stroke, and cardiac disease).

Two hundred three patients underwent esophagogastroduodenal endoscopy and/or ileocolonoscopy. The jejunal and ileal mucosae were visualized by contrast radiography, enteroscopy, and capsule endoscopy in 59, 29, and 37 patients, respectively. Table $\mathrm{S} 1$ gives the location and Fig. 1 shows the endoscopic appearance of gastrointestinal polyps. In this survey, in contrast to prior reports [10, 11], the esophagus was involved in 26 cases (12.3\%). Most of the esophageal biopsies revealed nonspecific inflammation, and some showed squamous papilloma of the esophagus. Compared with the stomach and colon, the jejunum and ileum were not as severely involved, mostly populated with sparsely distributed smaller polyps (less than $10 \mathrm{~mm}$ ), which are likely difficult to detect among the markedly inflamed mucosa. Gastric and colonic polyps were mostly sessile $(2-40 \mathrm{~mm})$ with engorged mucosa and rare punctate hemorrhages. Gastric polyps were smaller and more confluent than their colonic counterparts (Fig. S1). The useful distinguishing feature of juvenile polyps is abnormal-appearing edematous and reddish mucosa between polyps $[12,18]$, which was observed in the stomach and colon in 74 and $57.6 \%$ of cases, respectively.

Analysis of the histological results of 189 stomach biopsies and 197 colon biopsies revealed that nearly half of the gastric polyps $(52.4 \%$; 99 of 189$)$ and $57.9 \%$ of colonic polyps (114 of 197) displayed common features typical of CCS polyps such as focal dilated cystic glands, some filled with proteinaceous fluid or inspissated mucus, whereas the others displayed hyperplastic, inflammatory, or adenomatous features. Prominent infiltration of eosinophils, lymphocytes, and neutrophils was present in one third. Typically, the polyp and interpolyp area was edematous, with congestion and chronic inflammation of the lamina propria and submucosa, even though endoscopically the mucosa appeared normal (data not shown).

As shown in Table 1, 60 separate gastrointestinal tract carcinomas (19 gastric cancers and 41 colon cancers) were diagnosed either at initial presentation or during the observation period, with adenomas diagnose in 72 patients. Cancer or adenoma was limited to the stomach or colon, with no dysplastic lesions located in the esophagus or in the small intestine. In the colon, 36 CCS patients $(17.1 \%)$ adenomatous changes were diagnosed at the first endoscopy. No preferential localization of colon cancer was present in CCS patients, with advanced colon cancers evenly distributed throughout the large intestine (six ascending colon, one transverse colon, five sigmoid colon, five rectum, two multiple sites).

The common symptoms or prevalent physical findings, and laboratory data are shown in Table 2. Chronic diarrhea was reported in $78.3 \%$ of patients. Ectodermal changes were present in more than $70 \%$ of patients, with $91.3 \%$ having the single finding of alopecia, onychodystrophy, or skin hyperpigmentation (Fig. S1). Hyperpigmentation was described as lentigo-like to light to dark brownish macules, distributed on the palms and soles, upper extremities, face, and chest. Although rare, one of the important CCS complications is intestinal bleeding. Of 20 patients with gastrointestinal bleeding, two bled massively; one from an eroded gastric polyp, and the other from an advanced gastric cancer. Another major complication is intussusception: ten patients developed colo-colic intussusception due to a polyp serving as the intussuscipiens, with three requiring surgical therapy.

As shown in Table 2, a significant decrease in total protein and albumin was initially present, which improved after initial treatment. Fecal $\alpha_{1}$-antitrypsin clearance or ${ }^{99 \mathrm{~m}} \mathrm{Tc}$ albumin scintigraphy revealed a high prevalence of gastrointestinal tract protein loss. Of 113 patients screened for H. pylori, 61 (54.0\%) were positive, with successful eradication in 15 , with endoscopic remission or response attained in $8(53.3 \%)$, although one patient developed gastric cancer after successful eradication. Of the 19 CCS patients with gastric cancer (not included in the H. pylori screened subjects), two were negative for $H$. pylori, and six were positive.

Figure 2 depicts a flow diagram for the treatment and clinical course of the 210 patients. Nine patients did not 
Stomach

(A) Distribution and size of polyps

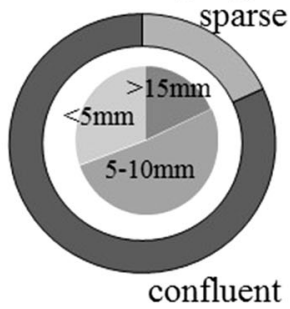

(B) Appearance of polyps

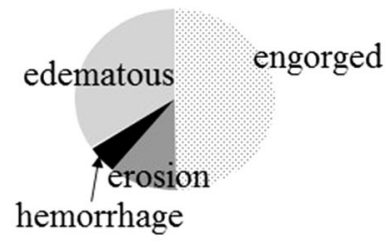

(C) Mucosal appearance between the polyps

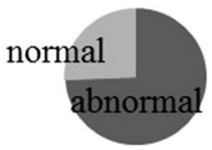

Fig. 1 Endoscopic findings of gastrointestinal polyps in patients with Cronkhite-Canada syndrome. A distribution and size of polyps. The outer circle shows the distribution pattern, and the inner circle shows the predominant size of the polyps. Data are expressed as the percentage of the total number of patients. The dominant types in the small intestine were sparsely distributed smaller polyps. The polyps in the stomach were more confluent than those in the colon $(81.9 \%$ vs $54.5 \%, p<0.01$ ), and smaller (in the stomach $18.1 \%$ were larger than $15 \mathrm{~mm}, 51.4 \%$ were $5-10 \mathrm{~mm}$, and $30.5 \%$ were smaller than
Small intestine
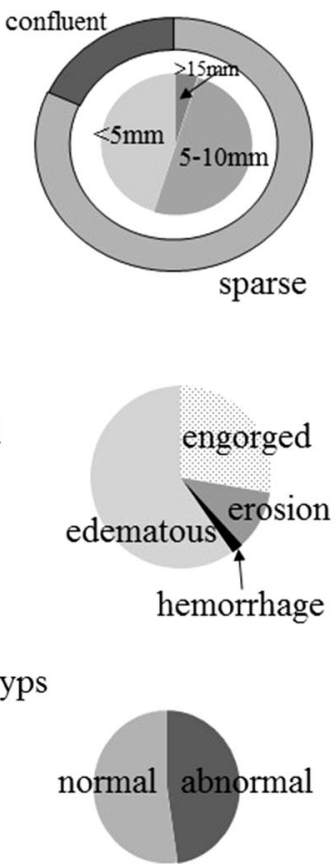

\section{Colon}

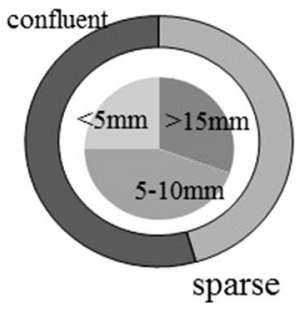

sparse
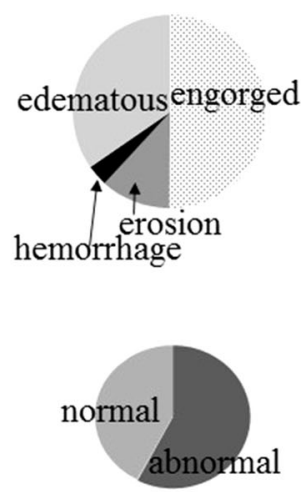

$5 \mathrm{~mm}$, and in the colon $30.4 \%$ were larger than $15 \mathrm{~mm}, 44.6 \%$ were $5-10 \mathrm{~mm}$, and $25 \%$ were smaller than $5 \mathrm{~mm} ; p<0.05$ ). $B$ endoscopic appearance of polyps. Appearance was classified into four categories (edematous, engorged, with erosion, or with hemorrhage) according to the most dominant appearance. $C$ the endoscopic appearance in the mucosa between the polyps was classified as normal or abnormal. Abnormal usually indicates edematous or reddish coarser mucosa than normal $(74 \%$ in the stomach and $57.6 \%$ in the colon, $p<0.01$ )

Table 1 Number of Cronkhite-Canada syndrome patients associated with gastric and colorectal cancer or adenoma

\begin{tabular}{|c|c|c|c|c|c|c|c|c|}
\hline & \multicolumn{6}{|l|}{ Cancer } & \multicolumn{2}{|l|}{ Adenoma } \\
\hline & \multicolumn{3}{|l|}{ Gastric } & \multicolumn{3}{|l|}{ Colon } & \multirow[t]{2}{*}{ Gastroduodenal } & \multirow[t]{2}{*}{ Colon } \\
\hline & Early & Advanced & Total & Early & Advanced & Total & & \\
\hline \multicolumn{9}{|l|}{ Associated patients } \\
\hline At initial presentation & $7(3.3 \%)$ & $6(2.9 \%)$ & $13(6.2 \%)$ & $11(5.3 \%)$ & $15(7.1 \%)$ & $26(12.4 \%)$ & $4(1.9 \%)$ & $36(17.1 \%)$ \\
\hline During observation & $2(1.0 \%)$ & $4(1.9 \%)$ & $6(2.9 \%)$ & $10(4.8 \%)$ & $5(2.4 \%)$ & $15(7.1 \%)$ & $3(1.4 \%)$ & $29(13.8 \%)$ \\
\hline Observation period (years) & $10 \pm 7.1$ & $11.7 \pm 7.0$ & $11.0 \pm 6.2$ & $8.8 \pm 4.6$ & $10.2 \pm 8.2$ & $9.2 \pm 5.9$ & $7.7 \pm 7.4$ & $6.4 \pm 4.8$ \\
\hline Total & $9(4.3 \%)$ & $10(4.8 \%)$ & $19(9.1 \%)$ & $21(10.1 \%)$ & $20(9.5 \%)$ & $41(19.5 \%)$ & $7(3.3 \%)$ & $65(31.0 \%)$ \\
\hline \multicolumn{9}{|l|}{ Therapeutic outcome } \\
\hline Endoscopic resection & & & 6 & & & 16 & 3 & 35 \\
\hline Surgery & & & 9 & & & 19 & 3 & 2 \\
\hline Death & & & 3 & & & 3 & 3 & 1 \\
\hline
\end{tabular}

Total of 210 patients. Adenomas bearing carcinomatous foci $(n=7)$, and adenomas that evolved into carcinoma $(n=28)$ were regarded as cancer. Three patients had both gastric and colon cancers, which were counted individually. All early colon cancers were found close to the adenoma. 
Table 2 Clinical variables: symptoms and laboratory findings in Cronkhite-Canada syndrome

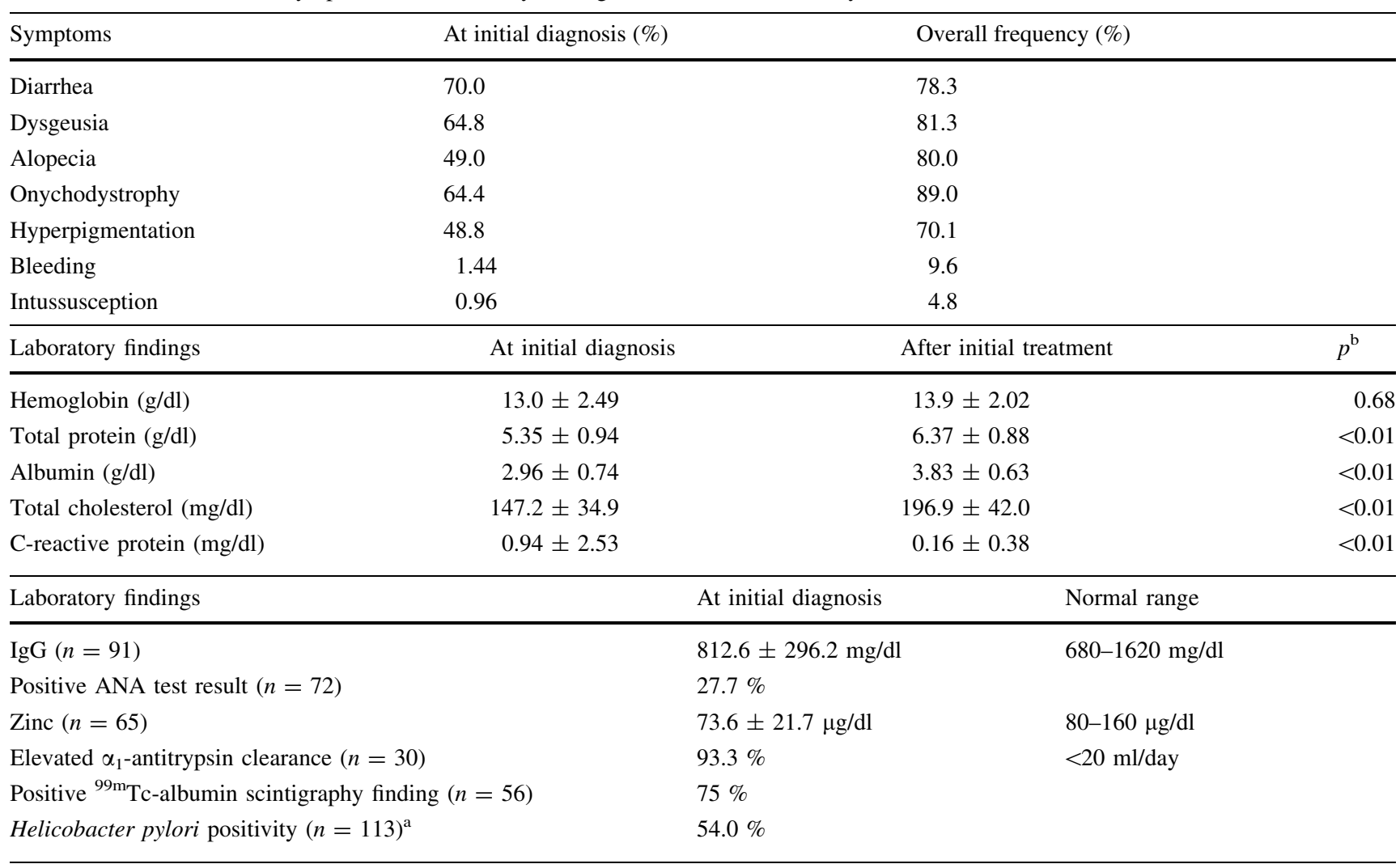

Total of 210 patients.

ANA antinuclear antibodies

${ }^{\text {a }}$ Diagnostic testing for $H$. pylori was performed with one of or a combination of rapid urease testing, culture, histology, IgG antibodies to $H$. pylori, or fecal antigen.

b Statistical comparison laboratory data between "at initial diagnosis" and "after initial treatment"

receive any intervention. Among them, three experienced spontaneous complete symptomatic remission without medication or nutritional therapy, sustained for more than 5 years. Among 201 treated patients, 178 received corticosteroid treatment, with the others were treated either with nutritional therapy or with surgical or endoscopic treatment alone. Treatment outcomes at 1 year were available for 173 patients: in the 23 patients who did not receive steroids, 21 were classified as having no early response, whereas a good early response was reported by $80.0 \%(120 / 150)$ of the group in whom corticosteroid therapy was initiated and continued for 1 year. Among 30 no early response cases, seven patients failed to respond who had progressive disease requiring intensive therapy, with $86 \%$ receiving intensive therapy, such as calcineurin inhibitors, octreotide, and anti-TNF- $\alpha$ agents, attaining a good early response. Among those who were treated with corticosteroids for 1 year, the subsequent outcome was evaluated in 108 patients over the following 3 years.

Nutritional therapy, including total parenteral nutrition and a defined-formula diet, frequently accompanied steroid therapy. Among 150 patients receiving corticosteroid treatment for 1 year, concurrent nutritional therapy was used in $68(45.3 \%)$, with 94 patients receiving nutritional therapy, including total parenteral nutrition and a definedformula diet. Yet, no significant difference in the good early response group was noted between groups receiving (90\%; 60 of 68 ) or not receiving (81\%; 61 of 75) nutritional therapy, suggesting no apparent additional beneficial effect of the nutritional therapy used.

Figure 3 shows the time course of clinical features by cumulative clinical remission rate after the induction of medical treatment. Remission of diarrhea occurred over 51 days, followed by reversal of dysgeusia ( 84 days) and ectodermal changes ( $97 \pm 11.2$ days). Decrease in the size and number of polyps was slower, averaging 248 days for gastric polyps and 238 days for colonic polyps.

Steroid therapy regimens differed among centers. Although dose escalation improved therapeutic outcomes, it was accompanied by severe side effects such as sepsis and thrombosis for dosages exceeding $60 \mathrm{mg} /$ day. Table S2 depicts the 1-year endoscopic response (good 
early response) stratified by initial dose. Since a greater than $85 \%$ response was observed with dosages exceeding $30 \mathrm{mg} /$ day, dosing at 30-49 mg/day appears optimal.

The subsequent course of patients treated with corticosteroids is shown in Fig. 4. Among 108 CCS patients who were initially treated with steroids and who were regularly evaluated for more than 3 years, $61.1 \%$ (66 of 108 ) had a long-term response, whereas $38.9 \%$ (42 of 108) had no long-term response. Of 66 patients who went into endoscopic remission (long-term response) after completion of an initial trial of steroid therapy, 36 patients $(54.5 \%)$ remained in endoscopic remission without further steroid therapy during the observation period (complete remission), and 30 patients (45.4\%) had sustained endoscopic remission with stable low-dose corticosteroid therapy (less than $10 \mathrm{mg}$; partial remission). Of the 42 patients who could not achieve sustained endoscopic remission (no long-term response), 16 (38.0\%) experienced frequent relapses after corticosteroid withdrawal (episodic active) and $26(61.9 \%)$ relapsed during tapering or for them steroid treatment could not be withdrawn (chronic active disease).

The ultimate aim of treatment is to prevent the development of CCS-related cancer. As shown in Fig. 4, sustained endoscopic improvement correlated with a significantly diminished risk of developing gastrointestinal cancer and CCS-related death due to complications. Among 66 patients with sustained endoscopic response (long-term response), only one case of cancer emerged, with no CSS-related death during the 3 years, whereas in the no long-term response group, a significantly higher incidence of cancer (17/42) and CSS-related death (7/42) was observed $(p<0.01)$.

After initial steroid treatment, immunomodulators or anti-TNF- $\alpha$ agents may possibly reduce or eliminate steroid use, preventing the recurrence of CCS-related polyps [5]. Of the five patients receiving azathioprine, two did not relapse during 2-11 years of monitoring, although three developed cancer with relapse of polyposis. Two patients who received a calcineurin inhibitor and one patient who received an anti-TNF- $\alpha$ agent remained in remission for 3 years. Nevertheless, in this survey, therapy with histamine $\mathrm{H}_{2}$ receptor antagonists or 5-aminosalicylate acid, which were taken by 28 or 46 patients, respectively, did not affect clinical or endoscopic outcomes or the risk of cancer when it was combined with other medications (data not shown).

Of 37 surgical operations performed in 36 patients, seven were for gastric cancer, 19 were for colon cancer, three were for gastroduodenal adenoma, one was for severe enteric protein loss, four were for intussusception, two were for gastric bleeding, and one was for gastric gastrointestinal stromal tumor. No operative complications were reported. The mean duration of posttreatment follow-up of the surviving patients was $5.1(0-14)$ years. Although symptomatic remission was attained in all cases, remission could not be attributed to the resection, since medical therapy, in particular steroid treatment, more likely contributed to the improvement.
Fig. 2 Flow diagram of treatment choice and clinical course of patients with Cronkhite-Canada syndrome $(C C S) * 1$ of 23 patients without steroid treatment, eight patients were treated with nutritional therapy and 15 patients underwent surgical/endoscopic treatment alone. $* 2$ among 30 patients who failed to respond to corticosteroid treatment, seven patients showing progressive disease required intensive therapy with calcineurin inhibitors $(n=4)$, octreotide $(n=1)$, anti-TNF- $\alpha$ agents $(n=1)$, and colectomy $(n=1)$. With the exception of one patient treated with a calcineurin inhibitor who failed to respond, the others attained endoscopic response
Total number of patients $(n=210)$

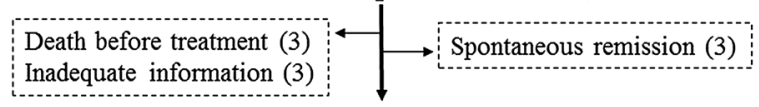

Treated cases $(n=201)$

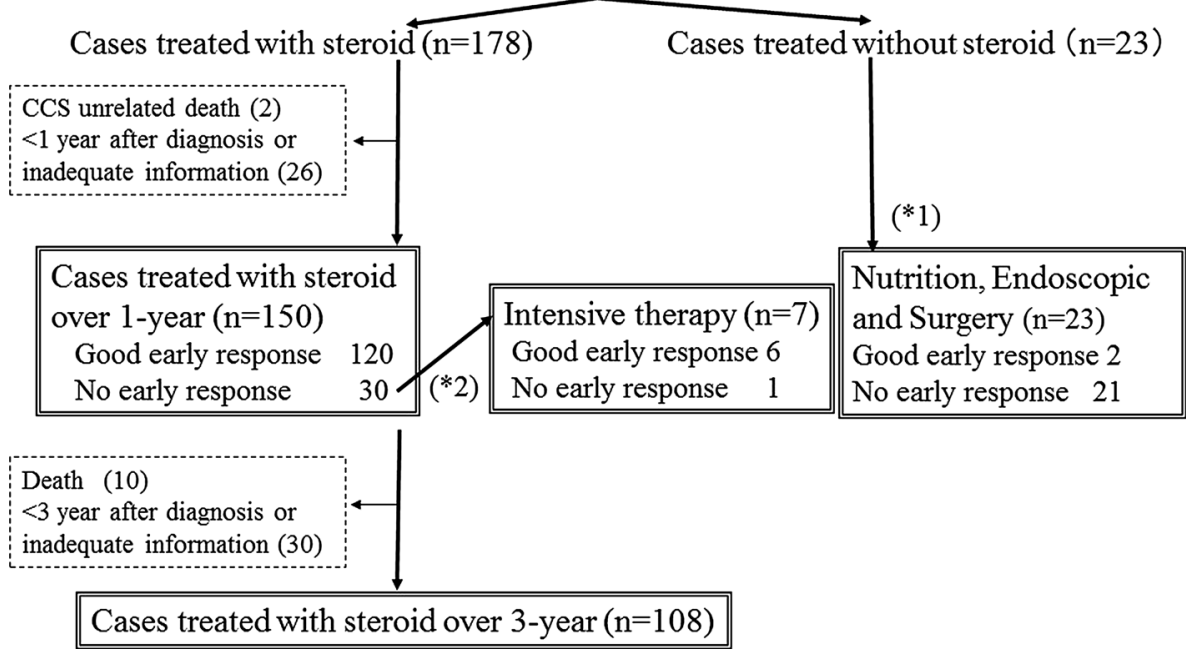



remission rate over time and mean time to remission. Asterisk the mean time to remission is significantly longer compared with the mean time diarrhea), dagger $p<0.05$ versus onychodystrophy, S.E. standard error
Fig. 3 The cumulative clinical for diarrhea $(p<0.05$ vs

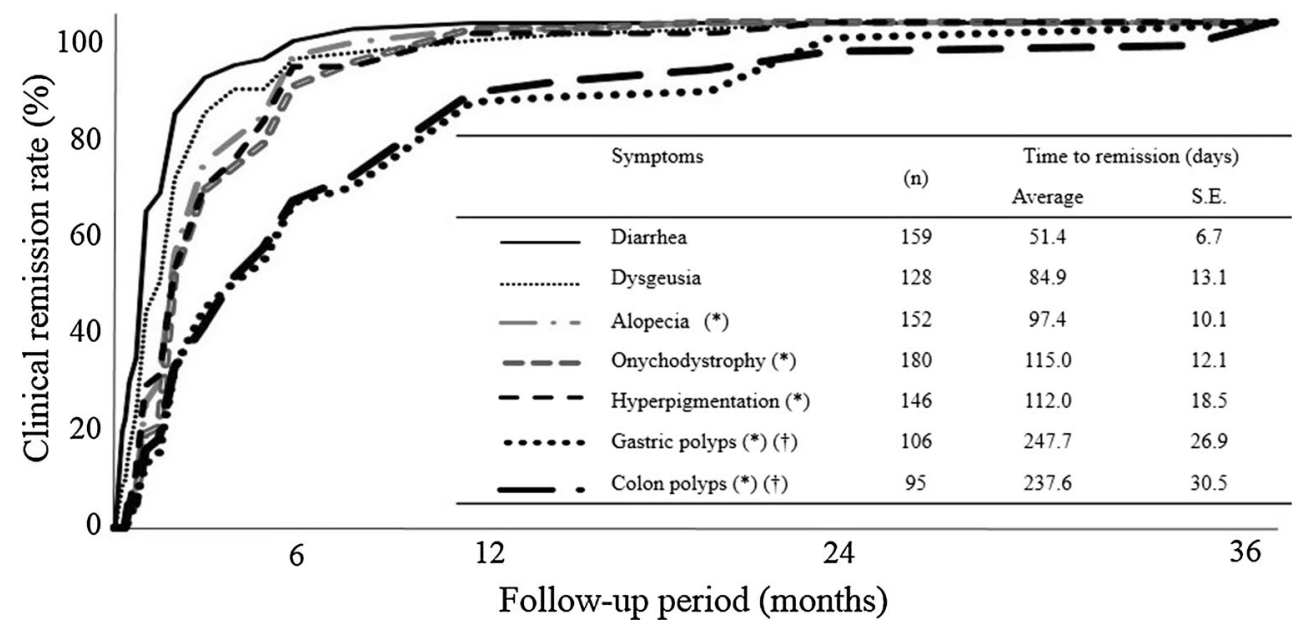

Fig. 4 Three-year outcome of steroid treatment in 108 patients who attained a good response. Of the 108 patients, 66 achieved a long-term response (36 "complete remission" and 30 "partial remission"), and 42 had no long-term response (16 "episodic active" and 26 "chronic active"). Among those patients, the occurrence of cancer (4 gastric cancer and 14 colon cancer) and CronkhiteCanada syndrome $(C C S)$-related death (one death due to sepsis caused by colitis, two deaths due to gastric cancer, and four deaths due to colon cancer) are shown

\begin{tabular}{|c|c|c|c|c|}
\hline \multirow{2}{*}{$\begin{array}{c}\text { Prolonged } \\
\text { outcome }\end{array}$} & \multicolumn{2}{|c|}{ Long-term response } & \multicolumn{2}{|c|}{ No long-term response } \\
\hline & $\begin{array}{l}\text { Complete } \\
\text { remission }\end{array}$ & $\begin{array}{l}\text { Partial } \\
\text { remission }\end{array}$ & $\begin{array}{l}\text { Episodic } \\
\text { active }\end{array}$ & $\begin{array}{l}\text { Chronic } \\
\text { active }\end{array}$ \\
\hline Steroid & - & & & \\
\hline $\begin{array}{l}\text { Endoscopic } \\
\text { findings }\end{array}$ & $\begin{array}{c}\text { Regression of } \\
\text { polyposis }\end{array}$ & $\begin{array}{c}\text { Regression of } \\
\text { polyposis }\end{array}$ & Polyposis & Polyposi \\
\hline $\begin{array}{c}\text { Number of } \\
\text { patients }\end{array}$ & 36 & 30 & 16 & 26 \\
\hline Cancer & 0 & 1 & 5 & 12 \\
\hline $\begin{array}{c}\text { Death } \\
\text { (CCS related) }\end{array}$ & 0 & 0 & 2 & 5 \\
\hline
\end{tabular}

\section{Discussion}

We investigated the clinical characteristics, response to medical treatment, and prognosis of 210 patients with CCS. Contrary to earlier reports [10,11], the prognosis of CCS has greatly improved through advances in medical treatment, although the course of CCS is relentlessly progressive, accompanied by an elevated cancer risk. Corticosteroid therapy, timely endoscopic evaluation, and the progress of medical management appear to improve the natural history of CCS. From our study, the diagnosis of CCS is confirmed by combination of clinical signs such as ectodermal abnormalities with characteristic foregut and hindgut endoscopic findings. Small bowel involvement, characterized as sparsely distributed small polyps, was difficult to detect radiologically. Histological diagnosis depends on the sample location, with the highest yield obtained from biopsies of the polyp and the adjacent mucosa. Since adenoma or early cancer commonly exists in the background of severe inflammation, it is difficult to endoscopically discriminate malignant polyps or concurrent adenocarcinoma among the numerous inflammatory CCS polyps.

Clinical manifestations followed a similar course in most patients, initially diarrhea, followed by ectodermal changes, which were at times attributed to malnutrition, since ectodermal changes improved with nutritional repletion. Lastly, inflammatory polyposis slowly regressed, taking as long as 240 days, indicating that long-term monitoring is required to assess treatment outcomes, even, in most cases, after remission of clinical symptoms. We thus strongly advocate continued endoscopic surveillance even after clinical remission has been achieved. 
Traditionally, steroid therapy and nutritional support are the mainstays of medical treatment $[1,11,17,24]$. We confirmed that a 30-49-mg daily dose of orally administered prednisolone was optimal for active CCS. A too-rapid steroid dose reduction can be associated with early relapse, suggesting that the prednisolone dose should be slowly tapered only after endoscopic confirmation of regression of polyposis. Poorer nutritional status at diagnosis may have increased the time to attain endoscopic remission with steroid therapy; therefore, those at higher risk from steroid therapy such as the elderly and those with a history of tuberculosis may benefit from nutritional support and consequent shortening of the length of steroid regimens. Steroid-sparing therapies such as cyclosporine A, octreotide, and an anti-TNF- $\alpha$ agent, which showed promise in a few cases, can be used in steroid-resistant cases in order to induce or maintain clinical remission. Although azathioprine used with or without steroid maintenance therapy was reported to be associated with sustained clinical remission $[1,5,32]$, we could not confirm this finding. Since immunological dysregulation is one of the important factors hypothesized to be present in CCS [5, 14, 33-36], the long-term use of immunoregulatory drugs or biologics may be useful for active or refractory disease.

One of our major findings was that achievement of sustained endoscopic remission was unambiguously associated with decreased cancer risk. Formerly, it was reported that total regression of polyps could not be attained even after long-term sustained clinical remission, with "atrophic" polyps observed [37]. Although histological confirmation is difficult, endoscopic documentation of regression of polyposis is likely to be an important primary therapeutic end point.

The increased incidence of gastric and colorectal carcinoma suggests the need for regular surveillance [17]. As previously mentioned, detection of malignancy at initial endoscopy is extremely difficult. Surveillance may be practical only after the diffuse inflammatory polyps have regressed with steroid therapy, which then reveals the otherwise "buried" adenomas or cancer, even in patients with sustained clinical remission. In four cases, advanced endoscopic imaging techniques, such as narrow-band imaging, magnifying endoscopy, and dye-based contrast-enhancement techniques, were helpful in identifying concurrent premalignant or malignant lesions that could be successfully resected, as previously reported (data not shown) [38, 39]. The surveillance interval thereafter is empiric, but as a minimum annual surveillance is recommended [1, 17], because in this survey we observed endoscopic recurrence in several cases before clinical recurrence approximately 1 year after discontinuation of corticosteroid therapy.

Another key question is the cause of the reversible ectodermal and gastrointestinal mucosal abnormalities characteristic of CCS. Although numerous factors have been suggested, such as genetic abnormalities [40], stress [41], immune dysregulation [5, 34, 35, 42, 43], and lowturnover cell differentiation [37, 44], no consensus has emerged, likely owing to the rarity of CCS. In our analysis, no familial occurrence was verified, one patient improved only after removal of stress, and another achieved prolonged clinical remission with nutrition therapy alone. Nevertheless, remission in the absence of specific therapies is difficult to distinguish from spontaneous improvement. As for immune disorders, only six cases were comorbid with immunological thyroid disease, one with rheumatoid arthritis, and 22 were low-titer antinuclear antibodies positive, inconsistent with an autoimmune cause [42]. There are several reports suggesting the strong association of CCS with IgG4-related disorders [5, 34], but in this study no examined serum sample was positive for IgG4, although the number of samples studied was low $(n=10)$. Moreover, no IgG4-positive plasma cells were reported in biopsy specimens (data not shown). Other proposed theories such as dysregulation of crypt cell differentiation and altered intestinal mucin production await experimental confirmation [14, 37, 44].

The risk of neoplasia remains controversial, with ongoing questions regarding the carcinogenic potential of the disease. Among 383 reported cases of CCS in Japan from 1980 to 2011, 40 patients (54 lesions; $10.2 \%$ ) with coincident gastric cancer and 51 patients (69 lesions) with coincident colon cancer were identified [7, 9, 22, 45]. In this study, the prevalence of gastric or colon cancer among CCS patients was approximately 10-20\%, similar to previous reports, both significantly higher than the prevalence of these cancers in the general Japanese population [46]. In our survey, more than $75 \%$ of gastric cancers were welldifferentiated adenocarcinoma, with $34.6 \%$ of polypoid lesions diagnosed as tubular adenoma. Furthermore, in the colon, seven cases of adenoma-bearing carcinomatous foci were detected, with all early colon cancers detected close to the adenoma. Although these histopathological findings are consistent with the adenoma-carcinoma sequence driving CCS-related malignancy, aberrant crypt maturation or chronic inflammation in the lamina propria may increase malignant potential via inflammation-induced mutation [4, 47].

This retrospective study not only describes the largest number of cases over the longest observation period of all analogous published studies, it is also the first study incorporating extensive clinical and endoscopic evaluations, underscoring how endoscopic remission influences the course of CCS. Nevertheless, these cases were collected exclusively from teaching hospitals in an effort to obtain the most accurate endoscopic information, possibly inducing selection bias. Another limitation was that the 
bulky questionnaire, intended to accumulate useful objective data, seemed sometimes difficult to answer thoroughly, resulting in the "inadequate cases." Because of the rarity of CCS, controlled trials for treatment are extremely difficult in CCS populations. Further prospective studies are need to gather further data regarding the effectiveness of steroid-sparing treatment, such as immunomodulators or biologics, for the induction of remission for severe disease [48], and for long-term maintenance.

In conclusion, appropriate medical therapy can alter the natural history of CCS. Oral corticosteroid therapy (30-49 mg/day) appeared to be effective for active CCS. Its benefits, including clinical improvement and polyp regression, are usually apparent within 12 months. Durable polyp regression (endoscopic remission) is associated with a markedly improved prognosis, with decreased cancer risk. Annual surveillance colonoscopy is recommended to assess mucosal disease activity and to remove adenomas and other premalignant mucosal lesions.

Acknowledgments This study was supported by a Grant-in-Aid for the Intractable Disease Project of the Ministry of Health, Labour and Welfare of Japan, and by Grants-in-Aid for Scientific Research from the Japanese Ministry of Education, Culture, Sports, Science and Technology. The authors thank the following institutions for replying to the questionnaires in the first and second surveys: Asahikawa Medical University, Sapporo Medical University Hospital, Hokkaido University Hospital, Kushiro Rosai Hospital, KKR Sapporo Medical Center, National Hospital Organization Hokkaido Medical Center, Sapporo Higashi Tokushukai Hospital, Sunagawa City Medical Center, Iwate Medical University, Tohoku University Hospital, Akita University Hospital, Nakadori General Hospital, Tokyo Medical University Ibaraki Medical Center, Jichi Medical University Hospital, Isesaki Municipal Hospital, Saitama Medical University Hospital, Dokkyo Medical University Koshigaya Hospital, Saiseikai Kawaguchi General Hospital, Saitama Medical Center Jichi Medical University, Sekishindo Hospital, Chiba University Hospital, Funabashi Municipal Medical Center, The University of Tokyo Hospital, Kyorin University Hospital, Keio University Hospital, Tokyo Medical University Hachioji Medical Center, Tokyo Women's Medical University Hospital, Nihon University Itabashi Hospital, NTT Medical Center Tokyo, The Cancer Institute Hospital of JFCR, Hiratsuka GI Hospital, Kitasato University Kitasato Institute Hospital, Sanno Hospital, Shiseikai Daini Hospital, Toho University Ohashi Medical Center, Mishuku Hospital, Kitasato University Hospital, Showa University Fujigaoka Hospital, St. Marianna University School of Medicine, Yokohama City Seibu Hospital, Yokosuka Kyosai Hospital, Saiseikai Yokohamashi Nanbu Hospital, Nihon Koukan Hospital, Yokohama Sakae Kyosai Hospital, Federation of National Public Service Personnel Mutual Associations, National Hospital Organization Shinshu Ueda Medical Center, Niigata City General Hospital, Kanazawa University Hospital, Public Central Hospital of Matto Ishikawa, Toyama Rosai Hospital, Gifu Municipal Hospital, Nagano Chuo Hospital, Toki General Hospital, Juntendo Hospital Shizuoka Hospital, Hamamatsu Medical Center, Hamamatsu South Hospital, Nagoya City University Hospital, Fujita Health University Hospital, Aichi Cancer Center Hospital, Japanese Red Cross Nagoya Daiichi Hospital, Kainan Hospital, Daiyukai General Hospital, Saiseikai Matsusaka General Hospital, Japanese Red Cross Society Nagahama Hospital, Mitsubishi Kyoto Hospital, Osaka City University Hospital, Osaka Medical College Hospital, Kinki University Hospital, Osaka
General Medical Center, Osaka Red Cross Hospital, Kitano Hospital, Osaka Saiseikai Nakatsu Hospital, Ishikiriseikikai Hospital, Sakai City Hospital, Yodogawa Christian Hospital, Maki Hospital, Kobe University Hospital, Hyogo College of Medicine, Takarazuka City Hospital, St.Mary's Hospital, Miki-Sanyo Hospital, Yamato Takada Municipal Hospital, Wakayama Medical University Hospital, Shimane University Hospital, Masuda Red Cross Hospital, Okayama University Hospital, Kawasaki Medical School Hospital, Hiroshima City Hiroshima Citizens Hospital, Hiroshima General Hospital of West Japan Railway Company, Hiroshima City Asa Hospital, Yamaguchi University Hospital, Tokuyama Central Hospital, Yamaguchi Red Cross Hospital, Tokushima Prefecture Naruto Hospital, Tokushima Red Cross Hospital, Uwajima City Hospital, Matsuyama Red Cross Hospital, National Hospital Organization Kochi National Hospital, Takamatsu Municipal Hospital, Kurume University Hospital, Kitakyushu Municipal Medical Center, Tagawa Hospital, Kyushu Central Hospital, Fukuoka Higashi Medical Center, Nagata Hospital, Wakamatsu Hospital, University of Occupational and Environmental Health, Japan, Saga University Hospital, Kumamoto University Hospital, Beppu Medical Center, Nakatsu Municipal Hospital, Nakagami Hospital, Kyushu University Graduate School of Medical Sciences, Yame General Hospital, Fukuoka Sanno Hospital, University of Miyazaki Hospital, Iizuka Hospital, Kyoto University, Sapporo-Kosei General Hospital, Sannno Hospital, Nanbu Medical Center/Nanbu Child Medical Center, Jikei University, Jikei University Kashiwa Hospital, Jikei University Katsushika Medical Center, Nagoya Handa City Hospital, Tsuchiura Kyodo General Hospital, Yokohama Municipal Citizen's Hospital, Sano General Hospital, and National Defense Medical College.

Conflict of interest The authors declare that they have no conflict of interest.

Open Access This article is distributed under the terms of the Creative Commons Attribution-NonCommercial 4.0 International License (http://creativecommons.org/licenses/by-nc/4.0/), which permits any noncommercial use, distribution, and reproduction in any medium, provided you give appropriate credit to the original author(s) and the source, provide a link to the Creative Commons license, and indicate if changes were made.

\section{References}

1. Sweetser S, Alexander GL, Boardman LA. A case of CronkhiteCanada syndrome presenting with adenomatous and inflammatory colon polyps. Nat Rev Gastroenterol Hepatol. 2010;7:460-4.

2. Elshout G, Dor FJ, Verhoog LC, et al. A Dutch patient presenting with a rare clinical syndrome. Cronkhite-Canada syndrome. Gut. 2011;60:1213.

3. Slavik T, Montgomery EA. Cronkhite-Canada syndrome six decades on: the many faces of an enigmatic disease. J Clin Pathol. 2014;67:891-7.

4. Seshadri D, Karagiorgos N, Hyser MJ. A case of CronkhiteCanada syndrome and a review of gastrointestinal polyposis syndromes. Gastroenterol Hepatol. 2012;8:197-201.

5. Sweetser S, Ahlquist DA, Osborn NK, et al. Clinicopathologic features and treatment outcomes in Cronkhite-Canada syndrome: support for autoimmunity. Dig Dis Sci. 2012;57:496-502.

6. Cronkhite LW Jr, Canada WJ. Generalized gastrointestinal polyposis; an unusual syndrome of polyposis, pigmentation, alopecia and onychotrophia. N Engl J Med. 1955;252:1011-5.

7. Matsui S, Kibi M, Anami E, et al. A case of Cronkhite-Canada syndrome with multiple colon adenomas and early colon cancers. 
Nippon Shokakibyo Gakkai Zasshi. 2011;108:778-86 (Article in Japanese).

8. De Petris G, Chen L, Pasha SF, et al. Cronkhite-Canada syndrome diagnosis in the absence of gastrointestinal polyps: a case report. Int J Surg Pathol. 2013;21:627-31.

9. Isobe T, Kobayashi T, Hashimoto K, et al. Cronkhite-Canada syndrome complicated with multiple gastric cancers and multiple colon adenomas. Am J Case Rep. 2013;14:120-8.

10. Goto A. (Cronkhite-Canada syndrome: epidemiological study of 110 cases reported in Japan). Nihon Geka Hokan. 1995;64:3-14.

11. Daniel ES, Ludwig SL, Lewin KJ, et al. The Cronkhite-Canada syndrome. An analysis of clinical and pathologic features and therapy in 55 patients. Medicine. 1982;61:293-309.

12. Calva D, Howe JR. Hamartomatous polyposis syndromes. Surg Clin North Am. 2008;88:779-817.

13. Burke AP, Sobin LH. The pathology of Cronkhite-Canada polyps. A comparison to juvenile polyposis. Am J Surg Pathol. 1989;13:940-6.

14. Jenkins D, Stephenson PM, Scott BB. The Cronkhite-Canada syndrome: an ultrastructural study of pathogenesis. J Clin Pathol. 1985;38:271-6.

15. Hashimoto K, Kashihara T, Kotani K, et al. A case of CronkhiteCanada syndrome with spontaneous regression. Dig Endosc. 1992;34:2615-20.

16. Viranuvatti V, Damrongsak C, Chainuvati T, et al. Cronkhite Canada syndrome: report of a case with spontaneous recovery. J Med Assoc Thai. 1981;64:261-6.

17. Ward EM, Wolfsen HC. Pharmacological management of Cronkhite-Canada syndrome. Expert Opin Pharmacother. 2003;4:385-9.

18. Ward EM, Wolfsen HC, Ng C. Medical management of Cronkhite-Canada syndrome. South Med J. 2002;95:272-4.

19. Yamaguchi K, Ogata Y, Akagi Y, et al. Cronkhite-Canada syndrome associated with advanced rectal cancer treated by a subtotal colectomy: report of a case. Surg Today. 2001;31:521-6.

20. Nagata J, Kijima $H$, Hasumi $K$, et al. Adenocarcinoma and multiple adenomas of the large intestine, associated with Cronkhite-Canada syndrome. Dig Liver Dis. 2003;35:434-8.

21. Yashiro M, Kobayashi $\mathrm{H}$, Kubo N, et al. Cronkhite-Canada syndrome containing colon cancer and serrated adenoma lesions. Digestion. 2004;69:57-62.

22. Egawa T, Kubota T, Otani Y, et al. Surgically treated CronkhiteCanada syndrome associated with gastric cancer. Gastric Cancer. 2000;3:156-60.

23. Malhotra R, Sheffield A. Cronkhite-Canada syndrome associated with colon carcinoma and adenomatous changes in C-C polyps. Am J Gastroenterol. 1988;83:772-6.

24. Ward EM, Wolfsen HC. The non-inherited gastrointestinal polyposis syndromes. Aliment Pharmacol Ther. 2002;16:333-42.

25. Russell DM, Bhathal PS, St John DJ. Complete remission in Cronkhite-Canada syndrome. Gastroenterology. 1983;85:180-5.

26. Takakura M, Adachi $\mathrm{H}$, Tsuchihashi N, et al. A case of Cronkhite-Canada syndrome markedly improved with mesalazine therapy. Dig Endosc. 2004;16:74-8.

27. Allbritton J, Simmons-O'brien E, Hutcheons D, et al. CronkhiteCanada syndrome: report of two cases, biopsy findings in the associated alopecia, and a new treatment option. Cutis. 1998;61:229-32.

28. Watanabe D, Ooi M, Hoshi N, et al. Successful treatment of Cronkhite-Canada syndrome using anti-tumor necrosis factor antibody therapy. Endoscopy. 2014;46(Suppl 1):476-7.
29. Okamoto $\mathrm{K}$, Isomoto $\mathrm{H}$, Shikuwa $\mathrm{S}$, et al. A case of CronkhiteCanada syndrome: remission after treatment with anti-Helicobacter pylori regimen. Digestion. 2008;78:82-7.

30. Chadalavada R, Brown DK, Walker AN, et al. Cronkhite-Canada syndrome: sustained remission after corticosteroid treatment. Am J Gastroenterol. 2003;98:1444-6.

31. Spigelman AD, Williams CB, Talbot IC, et al. Upper gastrointestinal cancer in patients with familial adenomatous polyposis. Lancet. 1989;2:783-5.

32. Ohmiya N, Nakamura M, Yamamura T, et al. Steroid-resistant Cronkhite-Canada syndrome successfully treated by cyclosporine and azathioprine. J Clin Gastroenterol. 2014;48:463-4.

33. Bettington M, Brown IS, Kumarasinghe MP, et al. The challenging diagnosis of Cronkhite-Canada syndrome in the upper gastrointestinal tract: a series of 7 cases with clinical follow-up. Am J Surg Pathol. 2014;38:215-23.

34. Riegert-Johnson DL, Osborn N, Smyrk T, et al. CronkhiteCanada syndrome hamartomatous polyps are infiltrated with IgG4 plasma cells. Digestion. 2007;75:96-7.

35. Takeuchi Y, Yoshikawa M, Tsukamoto N, et al. CronkhiteCanada syndrome with colon cancer, portal thrombosis, high titer of antinuclear antibodies, and membranous glomerulonephritis. J Gastroenterol. 2003;38:791-5.

36. Lin HJ, Tsai YT, Lee SD, et al. The Cronkhite-Canada syndrome with focus on immunity and infection. Report of a case. J Clin Gastroenterol. 1987;9:568-70.

37. Freeman K, Anthony PP, Miller DS, et al. Cronkhite Canada syndrome: a new hypothesis. Gut. 1985;26:531-6.

38. Horii J, Uraoka T, Kato J, et al. Usefullness of narrow band imaging system with magnification in diagnosis and treatment of colorectal adenoma in patients with Cronkhite-Canada syndrome. Dig Endosc. 2010;52:64-70.

39. Watari J, Morita T, Sakurai J, et al. Endoscopically treated Cronkhite-Canada syndrome associated with minute intramucosal gastric cancer: an analysis of molecular pathology. Dig Endosc. 2011;23:319-23.

40. Patil V, Patil LS, Jakareddy R, et al. Cronkhite-Canada syndrome: a report of two familial cases. Indian J Gastroenterol. 2013;32:119-22.

41. Murata I, Yoshikawa I, Endo M, et al. Cronkhite-Canada syndrome: report of two cases. J Gastroenterol. 2000;35:706-11.

42. Qiao M, Lei Z, Nai-Zhong H, et al. Cronkhite-Canada syndrome with hypothyroidism. South Med J. 2005;98:575-6.

43. Anderson RD, Patel R, Hamilton JK, et al. Cronkhite-Canada syndrome presenting as eosinophilic gastroenteritis. Proc Bayl Univ Med Cent. 2006;19:209-12.

44. Watanabe-Okada $\mathrm{E}$, Inazumi $\mathrm{T}$, Matsukawa $\mathrm{H}$, et al. Histopathological insights into hair loss in Cronkhite-Canada syndrome: diffuse anagen-telogen conversion precedes clinical hair loss progression. Australas J Dermatol. 2014;55:145-8.

45. Karasawa H, Miura K, Ishida K, et al. Cronkhite-Canada Syndrome complicated with huge intramucosal gastric cancer. Gastric Cancer. 2009;12:113-7.

46. Ministry of Health Law. Vital statistics Japan. 2010.

47. Sweetser S, Boardman LA. Cronkhite-Canada syndrome: an acquired condition of gastrointestinal polyposis and dermatologic abnormalities. Gastroenterol Hepatol. 2012;8:201-3.

48. Martinek J, Chvatalova T, Zavada F, et al. A fulminant course of Cronkhite-Canada syndrome. Endoscopy. 2010;42(Suppl 2):350-1. 